\title{
WRKY Gene Silencing Enhances Tolerance to Salt Stress in Transgenic Tomato
}

\author{
Mengistu Wubie Birhanu* Christos Kissoudis C. G. van der Linden \\ Plant Breeding, Wageningen University \& Research, Wageningen, Netherlands
}

\begin{abstract}
WRKY proteins are superfamily of transcription factors, which are up or down-regulated in response to biotic and abiotic stress though limited study has conducted on transcriptional repression of tomato WRKY genes to salt stress. Thus, this study was conducted on three transgenic tomato (Solanum lycopersicum L. cv. Moneymaker) T1 generation plants carrying RNAi constructs for WRKY5, WRKY12, and WRKY13 to identify WRKYs that can act as negative regulators and uncover associated gene expression. In vitro propagated transgenic and wild type (MM) seedlings were transferred to pots under greenhouse. The plants were watered with standard solution supplemented with 0 or $100 \mathrm{mM} \mathrm{NaCl}$ for 4 weeks and evaluated for their responses. The result showed WRKY12 and WRKY13_RNAi lines performed better under salt stress with successful silencing which suggest the two WRKY transcription factors act as negative regulators. SOS1 is up regulated in WRKY13_RNAi, which may lead to low $\mathrm{Na}^{+}$accumulation and contribute to salt tolerance. Expression of APX is up regulated in WRKY12 and WRKY13_RNAi, which may contribute to salt tolerance mechanism of the two genotypes. Microarray analysis of abiotic stress related genes might give clear indication for the regulatory role of WRKY12 and WRKY13 in abiotic stress tolerance.
\end{abstract}

Keywords: Solanum lycopersicum. Salt Stress. WRKY_RNAi. Negative Regulator

DOI: $10.7176 / \mathrm{JBAH} / 10-17-03$

Publication date:September $30^{\text {th }} 2020$

\section{INTRODUCTION}

Transcription factors are proteins which have substantial role in regulating stress-inducible gene expression enabling plants to adapt biotic and abiotic stresses (Besseau et al., 2012; Chen et al., 2012; Gao et al., 2007; Singh et al., 2002). Transcription factors including dehydration responsive element-binding factor (DREB), ABAresponsive elements (ABARE), zinc-finger proteins, WRKY, and NAC transcription factors are induced by certain abiotic stresses and activate expression of abiotic stress related genes (Hu and Xiong, 2014). Overexpression or suppression of these regulatory genes enhances activation of different stress responsive genes (Zheng et al., 2009; Abe et al., 2003; Orellana et al., 2010). WRKY proteins are a superfamily of transcription factors containing one or two highly conserved WRKY domains, 60 amino acid region comprising conserved WRKYGQK sequences at $\mathrm{N}$-terminal together with zinc-finger-like motif at its C-terminal (Eulgem et al., 2000). They are known to be involved in regulating diverse functional processes such as growth, development, hormone-mediated pathways, biotic and abiotic stresses (Ramamoorthy et al., 2008). The WRKY transcription factors comprise different family members in different plant species. Studies indicated that there are 74 members of WRKY genes in Arabidopsis (Arabidopsis thaliana), 64 in soybean (Glycine max), 81 in tomato, 102 and 98 in indica and japonica rice, respectively (Huang et al., 2012; Ross et al., 2007; Ülker and Somssich, 2004; Zhou et al., 2008).

Based on the genome wide investigation of WRKY genes in tomato, a total of 81 WRKY members have been identified (Huang et al., 2012). Microarray analysis of these members showed significant change in expression pattern in response to biotic and abiotic stresses. Further expression analysis of selected SIWRKY in real-time quantitative RT-qPCR showed that majority of the tested SIWRKY genes were up-regulated in response to drought, salt, and pathogen invasion, while down regulation has also observed for some of the SIWRKY genes. Like other TFs, tomato WRKY TFs may act as transcriptional repressors. However, there is limited study on tomato either individual WRKY genes have transcriptional repression in stress response including salt stress. Thus, targeted down regulation of gene expression is an alternative approach to release suppression effect. RNA interference (RNAi) is a powerful tool that can aid to uncover the function of individual gene (Hannon, 2002). To study the involvement of WRKY genes in salt tolerance, several different WRKY genes were silenced in tomato through RNAi in transgenic lines that have been developed previously. Therefore, the objectives of this research were (1) to verify the silencing level of WRKY genes in transgenic tomato lines (2) to evaluate the phenotypic, physiological, and biochemical response of WRKY_RNAi transgenic lines to salt stress and (3) to identify WRKY genes that can act as negative regulators and uncover associated gene expression in response to salt stress.

\section{MATERIALS \& METHODS}

Transgenic plant materials

Transgenic tomato (Solanum lycopersicum L. cv. Moneymaker) T1 generation plants carrying RNAi constructs for WRKY gene silencing (WRKY5_RNAi, WRKY12_RNAi and WRKY13_RNAi) were used for the study. The 
RNAi transgenic tomato lines were propagated in vitro to perform subsequent experiments.

\section{Growth conditions}

Seeds harvested from first generation (T1) transgenic plants were cultured on MS agar medium supplemented with $100 \mathrm{mgL}^{-1}$ Kanamycin $(\mathrm{Kn})$. After 2 weeks of culture, kanamycin-resistant and kanamycin-sensitive seedlings were identified. Seedlings maintained on MS medium containing kanamycin were chosen as transformed plants while non-germinated were excluded. The transformants were grown and propagated further in vitro without Kn for subsequent experiments. The propagation was done by cutting the nodal part and growing on $1 / 2$ MS agar medium. For transfer to the greenhouse, the apical nodal part was cut and rooted on $3 / 4$ MS medium supplemented with 20 $\mathrm{mgL}^{-1}$ of cefotaxime as an antibacterial agent. After two weeks of in vitro growing, the seedlings were transferred to pots filled with vermiculite under greenhouse condition with a photoperiod regime of $16 \mathrm{hr}$ light and $8 \mathrm{hr}$ dark, and $70 \% \mathrm{RH}$.

\section{Treatment of salt stress}

The transferred seedlings were covered with a transparent cup for the first four days to acclimatize. The plants were further grown for 14 days by watering with half strength Hoagland nutrient solution. Then, the plants (4 plants per line) were watered with standard solution supplemented with 0 or $100 \mathrm{mM} \mathrm{NaCl}$ in one day interval for 4 weeks to evaluate their response to salt stress. Salt solution was applied till leaching was observed from the pot to maintain the appropriate and uniform concentration.

\section{Experimental design and evaluation of salt tolerance}

The experiment was laid out in randomized complete block design with two treatments (control and salt) and four replications for each, consisting of the different WRKY_RNAi and wild type (MM) tomato plants.

\section{Morphological measurements}

Growth parameters such as shoot length, fresh weight and dry weight were measured at the end of the experiment (4 weeks after salt treatment). The above ground parts were harvested and the fresh weight (FW) was measured immediately after harvest. Samples were dried in oven at $70^{\circ} \mathrm{C}$ for $48 \mathrm{hrs}$ and dry weight (DW) was measured subsequently.

\section{Physiological assessment Chlorophyll content}

Chlorophyll content was measured 4 weeks after salt treatment using Minolta chlorophyll meter SPAD-502. Two measurements per plant were taken from fully expanded matured leaflets and the average value was taken for each individual plant.

\section{Electrolyte leakage}

Leaf samples were collected from the transgenic and wild type control plants. Twelve (12) leaf disks $(\sim 7 \mathrm{~mm}$ in diameter) were prepared from each line by cutting with metal leaf borer and put in tube containing $20 \mathrm{ml}$ of MQ ultrapure water supplemented with $1 \mu \mathrm{M}$ paraquat solution. The samples were incubated overnight under dark condition for $12 \mathrm{hrs}$. Then, the tubes were transferred to light condition for $24 \mathrm{hrs}$. The electrical conductivity of the solution was measured twice i.e. on $24 \mathrm{hrs}$ and $48 \mathrm{hrs}$ using EC meter. Then, samples were autoclaved at $121^{\circ} \mathrm{C}$ for $5 \mathrm{~min}$ and the final electrical conductivity was measured after cooling down. The electrolyte leakage (EL \%) was calculated in percentage as described in previous study (Dionisio-Sese and Tobita, 1998) using the equation; $\mathrm{EL}=\mathrm{ECi} / \mathrm{ECf} * 100$ Where, ECi: initial electrical conductivity (24 \& 48hrs); ECf: final electrical conductivity (after autoclaving).

\section{Biochemical analysis}

Dried leaves and stems were used for ion content analysis $\left(\mathrm{Na}^{+}, \mathrm{K}^{+}, \mathrm{Ca}^{2+}, \mathrm{Mg}^{2+}, \mathrm{SO}_{4}{ }^{2-}, \mathrm{PO}_{4}{ }^{3+}\right.$, and $\left.\mathrm{Cl}^{-}\right)$. The dried samples were ground with a $1 \mathrm{~mm}$ mesh. $29-31 \mathrm{mg}$ of the resulting powder was weighed and ashed in an oven for $6 \mathrm{hrs}$ at a maximum temperature of $575^{\circ} \mathrm{C}$. After cooling down, $1 \mathrm{ml}$ of $3 \mathrm{M}$ formic acid was added and the samples were shaken for $15 \mathrm{~min}$ at $99^{\circ} \mathrm{C}$ and $400 \mathrm{rpm}$ to dissolve the ash. $9 \mathrm{ml}$ of milliQ water was subsequently added and the solution was mixed properly. After that $200 \mu \mathrm{l}$ was taken from the mixture and added into ion chromatography (IC) plastic tube containing $9.8 \mathrm{ml}$ of milliQ water (50-fold dilution). The standards and blanks were prepared before the analysis. Ion content was measured with ion chromatography (Metrohm). Anions were analyzed by Methrom 881 compact IC pro (2.882.0020) using Metrosap A 150, 150/4.0 mm column equipped with a Mertosap C5/5 Supp 4/6 guard column. While, cations were analyzed by Methrom 881 Compact IC pro (2.882.0010) using Metrosap C4 Supp 4,250/4.0 mm column equipped with a Metrosap A Supp 4/6 guard column. The peak areas for the cations and anions were determined and converted to concentration according to the standard formula. Then, 
the ion content in leaves and stems was normalized to dry weight of the samples.

\section{Gene expression analysis \\ Primer design}

WRKY gene specific primers (Table 1) were designed using the available web-based primer design program (http://www.ncbi.nlm.nih.gov/). Primers were designed outside the RNAi construct towards 3' end of the sequence region. Multiple alignments were made for all the WRKY genes to identify non-conserved regions of the coding sequence to be used for primers design.

Table 7. Primer sequences used for the expression analysis of WRKY genes

\begin{tabular}{lll}
\hline WRKY gene & Primer name & Sequence ( 5'----> 3' order) \\
\hline WRKY5 & WRKY5_F & AGACCAGCAAAGAAATCTCCA \\
& WRKY5_R & TTTCTCCAGAAACACTTATGATCG \\
WRKY12 & WRKY12_F & GCATGAAAATGGCTGATATTCCACC \\
& WRKY12_R & AGCTGCATCGTCTAATGCTCTTT \\
WRKY13 & WRKY13_F & ACCATCACTCTTCTTCTTCTTCACC \\
& WRKY13_R & GAACTTGCAAAGGGGAATTGATGA \\
\hline
\end{tabular}

\section{RNA isolation \& cDNA synthesis}

Leaf samples were taken from the second leaf counting from the top (first to be moderately expanded) of salt treated and control plants. The samples were immediately frozen in liquid nitrogen and stored at $-80^{\circ} \mathrm{C}$ before RNA extraction. The leaf samples were ground thoroughly in liquid nitrogen with mortar and pestle. RNA was isolated using RNeasy Plant Mini Kit following the manufacturer's instruction. The concentration and quality of the isolated RNA was checked in nanodrop spectrometer and 2\% agarose gel electrophoresis, respectively. One $\mu \mathrm{g}$ of total RNA was treated with RNase-free DNase I (invitrogen) to prevent contamination with DNA. DNase I was inactivated with $1 \mu \mathrm{l}$ of $25 \mathrm{mM}$ EDTA solution. The RNA was then reverse transcribed using iScriptTM cDNA synthesis kit following the manufacturer's instruction.

\section{Quantitative real-time PCR (qPCR)}

After cDNA synthesis, real-time qPCR was used to examine and quantify the expression level of the WRKY genes targeted for silencing in each of the WRKY RNAi lines under control conditions. The reaction mix containing $5 \mu 1$ SYBR GREEN super mix, $1 \mu 1$ forward primer, $1 \mu 1$ reverse primer, and $3 \mu 1$ template cDNA was prepared with a final volume of $10 \mu \mathrm{l}$. The PCR amplification was set as initial denaturation at $95^{\circ} \mathrm{C}$ for 5 minutes followed by 39 cycles of $94^{\circ} \mathrm{C}$ for $10 \mathrm{~s}, 59^{\circ} \mathrm{C}$ for $30 \mathrm{~s}$ followed by a melt curve analysis. Relative expression of the genes was determined from the difference in cycles observed to reach the threshold $(\Delta \Delta \mathrm{Ct})$ between the target genes and reference gene. Elongation factor (EF-1a) was used as a reference gene in each PCR reaction. Each sample analysis was performed in two biological and two technical replicates. The expression level relative to the reference gene expression level was calculated using the formula; $2^{-\Delta \Delta \mathrm{Ct}}$ (Livak and Schmittgen, 2001).

\section{Expression analysis of stress related genes}

The expression of putative candidate genes involved in hormonal biosynthesis (NCED1: Absisic Acid Pathway), ion homeostasis (SOS1), cell death regulation (MCA1), and ROS scavenging genes (APX1 and SOD) was analyzed with real-time qPCR.

Table 8. . Primer sequences used for the expression analysis of stress related genes

\begin{tabular}{lll}
\hline Stress related genes & Primer name & Sequence (5'----> 3') \\
\hline SOD & SISOD_F & CCTCTCACTGGTCCACAGTCCA \\
& SlSOD_R & AGCAGTTAACCCTGGAGGCCA \\
APX1 & SlAPX1_F & CCATTTGGAACAATCAGGCACCCG \\
& SlAPX1_R & CGGGGCCTCCCGTAACTTCA \\
NCED1 & NCED1_F1 & TCGAAAACCCGGATGAACAAGTGA \\
& NCED1_R1 & AACCAGAAACTTTTGGCCATGGTTC \\
SOS1 & SlSOS1_F & GCACATCTTCACGATGCTGTCCA \\
& SISOS1_R & AGAGGGTTGCACCACGTAACTTCA \\
MCA1 & MCA_F1 & CACTCTTTGACGTCTTTGGCG \\
& MCA_R1 & AACCATACCCATGAACCCGC \\
\hline
\end{tabular}

\section{Statistical analysis}

The collected growth and physiological data were subjected to GenStat 16th edition (64 bit). Significance effects of the treatment were determined by analysis of variance (ANOVA). The treatment means were compared by Fisher protected least significance difference (LSD) test with a significance level of $\mathrm{p}<0.05$. Correlation analysis 
was made between morphological and biochemical parameters.

\section{RESULT}

\section{Expression Analysis of WRKY Genes}

Real-time PCR was performed using gene specific primers to determine the silencing level of WRKY genes in RNAi transgenic lines. The expression of individual WRKY genes in RNAi lines was checked only in control condition since we do not know their expression before and better to start from control. Their expression was calculated relative to the expression of the native WRKY genes in MM background. As expected, the relative expression of WRKY genes was lower compared to their expression in MM (Table 3).

Table 9. WRKY gene expression in independent RNAi lines ( $2^{\text {nd }}$ column) and wild type $(\mathrm{MM})$ background ( $4^{\text {th }}$ column) and the relative expression ( $5^{\text {th }}$ column) calculated by dividing expression of WRKYs in RNAi lines to their respective expression in MM background, $(\mathrm{n}=2)$.

\begin{tabular}{lllll}
\hline WRKY RNAi lines & Expression & SD & MM & Relative to MM \\
\hline WRKY5-2 & 0.0212 & 0.0072 & 0.0529 & 0.4016 \\
WRKY5-3 & 0.0074 & 0.0006 & 0.0529 & 0.1403 \\
WRKY12-1 & 0.1063 & 0.0192 & 0.2028 & 0.5239 \\
WRKY12-2 & 0.0218 & 0.0019 & 0.2028 & 0.1073 \\
WRKY12-3 & 0.0086 & 0.0011 & 0.2028 & 0.0424 \\
WRKY13-1 & 0.0002 & 0 & 0.0011 & 0.1821 \\
WRKY13-2 & 0.0002 & 0 & 0.0011 & 0.1681 \\
WRKY13-3 & 0.0003 & 0.0001 & 0.0011 & 0.2913 \\
\hline
\end{tabular}

$\mathrm{SD}=$ standard deviation

\section{Growth Response of Genotypes to Salt Stress}

Salt stress tolerance of RNAi lines silenced for 3 tomato WRKY genes and wild type (MM) tomato plants was evaluated under $100 \mathrm{mM} \mathrm{NaCl}$ and control conditions. Since the plants were vegetatively propagated, the transgenic lines were uniform while transferred to the greenhouse. Clear phenotypic differences were visible after two weeks of salt treatment. In addition to genotypic variation to salt stress response, some of the transgenic lines showed altered phenotype in control conditions. The WRKY5-3_RNAi transgenic plants showed wilted and yellowish leaves (cell death) in control condition but this was partly reversed under salt stress (picture not shown).

\section{Influence of Salinity on Shoot Length}

Shoot length was measured twice, i.e. two and four weeks after salt treatment. No significant differences in shoot length were found among genotypes in the first two weeks of salt treatment (data not shown). Visible phenotypic variation in shoot length was observed after the plants were exposed for four weeks to $100 \mathrm{mM} \mathrm{NaCl}$ treatment. The results indicate that salt stress significantly $(\mathrm{p}<0.05)$ decreased the shoot length of tomato genotypes as compared to control condition. The genotype WRKY12-1, and WRKY12-2 RNAi lines exhibited the highest shoot length under salt stress (Fig. 1a)

\section{Genotypic Variation in Biomass Production under Salt Stress}

To evaluate the effect of salt stress on the whole plant level, shoot fresh weight (SFW) and shoot dry weight (SDW) was calculated. Under salt stress WRKY12-1, WRKY12-2, WRKY13-1, and WRKY13-3 RNAi showed higher SFW, ranging from 236 to 264g, compared to MM and other lines (Fig. 1b), while WRKY5-3 RNAi had lower SFW both under salt and control conditions. Similar results were observed in shoot dry weight (SDW). Salt stress caused reduction in SDW compared to control (Fig. 1c). Under salt stress WRKY12-1 and WRKY13-3 RNAi lines showed higher SDW, while WRKY5-3 had lower SDW compared to MM and other lines. 

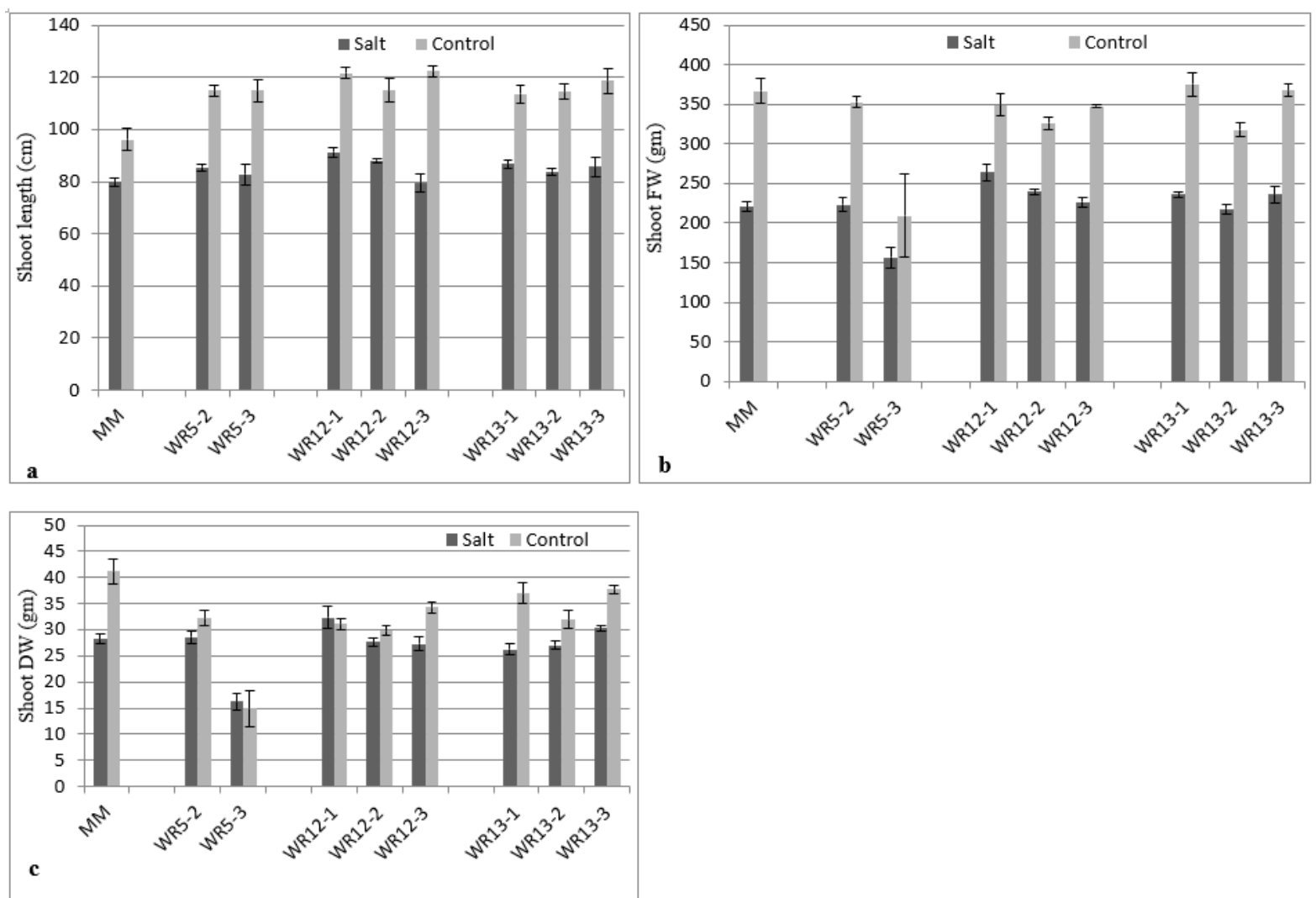

Figure 1. Growth response of WRKY-RNAi and wild type (MM) tomato under $100 \mathrm{mM} \mathrm{NaCl} \&$ control conditions. (a) Shoot length (cm), (b) Shoot fresh weight (gm), (c) Shoot dry weight (gm). Growth parameters were measured 4 weeks after salt treatment. ( $n=4$, error bars indicate the standard error)

\section{Physiological Response of Genotypes under Salt Stress Chlorophyll Content}

Chlorophyll content of the leaves can be used as an indicator of the photosynthetic activity and performance of the plants under stress conditions (Wu et al., 2008). Thus, tolerant plants are generally expected to have higher chlorophyll content than sensitive ones. In this study, chlorophyll content of transgenic and wild type plants was measured three weeks after $100 \mathrm{mM} \mathrm{NaCl}$ treatment. The result indicates that there was significant difference $(\mathrm{p}<0.05)$ in chlorophyll content among genotypes both under salt and control conditions. In general, the chlorophyll content of all the genotypes was higher under salt stress condition compared to control (Fig. 2d). Under salt stress higher chlorophyll content was measured in WRKY13-3 and WRKY12-3_RNAi plants with 61.32 and 61 SPAD readings, respectively compared to $\mathrm{MM}$ and other lines. In contrast, low chlorophyll content was measured in WRKY5-3 with 50.7 SPAD readings. The Chlorophyll content was slightly higher in MM (53 SPAD readings) under salt stress though not significantly different from most RNAi lines.

\section{Response of genotypes to oxidative stress}

Paraquat, a non-selective herbicide, acts as photosynthesis inhibitor. In many scientific studies paraquat is used as a model chemical to induce reactive oxygen species (ROS) (Bus and Gibson, 1984). Under light conditions, it accept electrons from photosystem-I (PS-I) and transfer them to oxygen molecules, inducing the production of ROS which induce injury to plant cells. Electrolyte leakage is an indication of plant membrane stability and can give indication to many stress related physiological and biochemical responses of plants including antioxidant enzyme synthesis, lipid peroxidation, and leaf senescence (Liu and Huang, 2000). In this study, membrane stability of the leaves against oxidative stress was investigated by measuring the electrolyte leakage percentage (EL \%) at two time point after incubating the leaf discs from transgenic and wild type plants in $1 \mu \mathrm{M}$ paraquat solution. As a result, significant differences in EL\% were found among the transgenic lines both 24 and $48 \mathrm{hrs}$ exposure time. Generally, electrolyte leakage increased drastically as the duration of the paraquat treatment increased from 24 to 48hrs (Fig. 2c). WRKY5-3_RNAi plants exhibited higher EL (53.9\%) compared to MM. The leaf discs of some transgenic lines were almost completely damaged after $48 \mathrm{hrs}$ though variation was found among genotypes. The EL \% was approximately doubled at $48 \mathrm{hrs}$ exposure as compared to $24 \mathrm{hrs}$, which suggest the cell membrane is severely damaged due to prolonged oxidative stress. WRKY5-2 and WRKY5-3 lines were sensitive to oxidative 


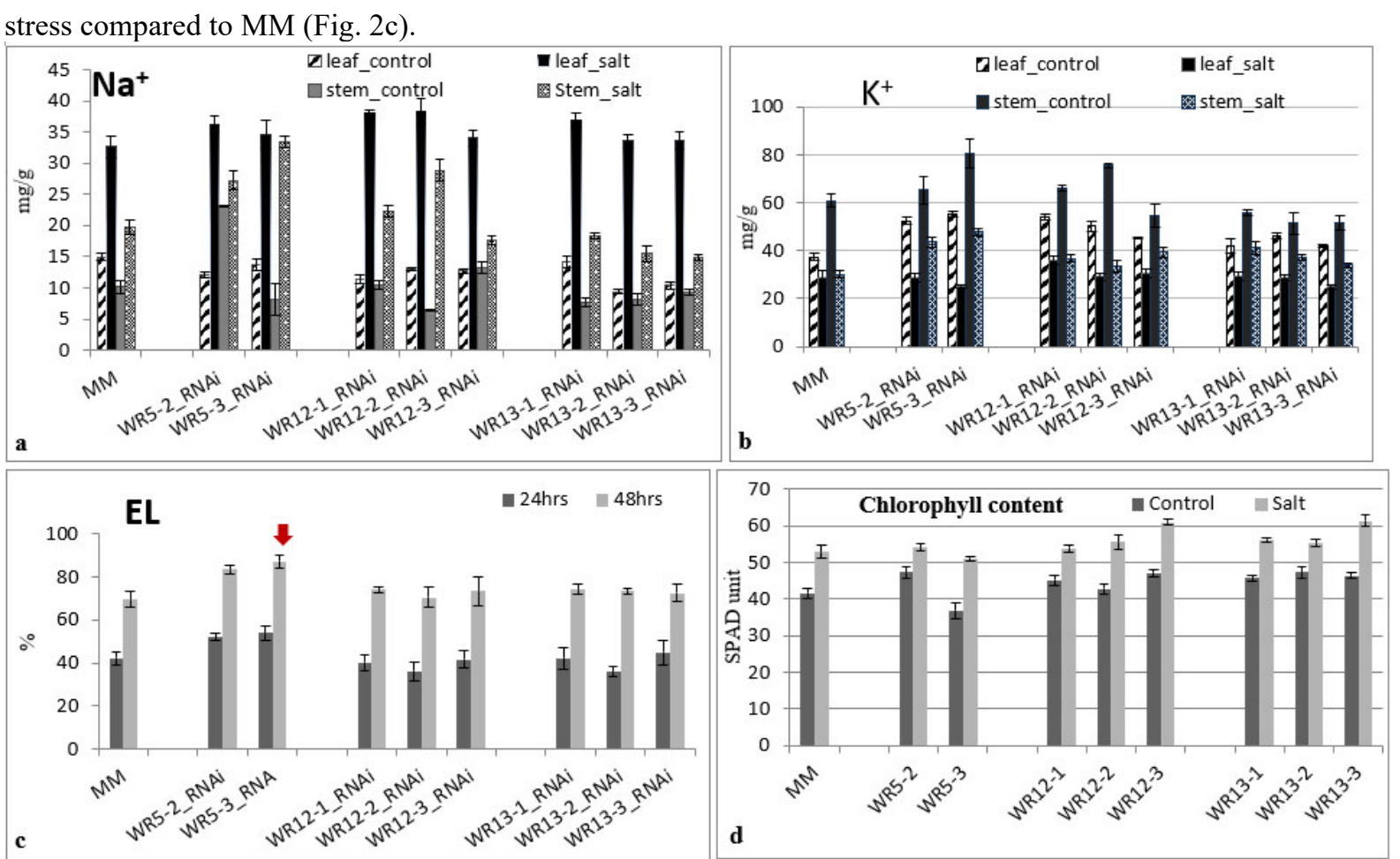

Figure 2. Biochemical (leaf \& stem ion content) \& physiological response of WRKY transgenic and wild type (MM) tomato plants under $100 \mathrm{mM} \mathrm{NaCl} \&$ control conditions. (a) Leaf and stem sodium $\left(\mathrm{Na}^{+}\right)$content $(\mathrm{mg} / \mathrm{g})$, (b) Leaf and stem potassium $\left(\mathrm{K}^{+}\right)$content $(\mathrm{mg} / \mathrm{g})$, (c) Electrolyte leakage (EL\%) after incubating the leaf discs for 24 and 48 hrs., (d) Chlorophyll content (SPAD unit), ( $n=4$, error bars represent standard error.

\section{Ion Content Analysis}

Leaf and stem ion content was determined in transgenic and wild type plants grown under salt and control conditions. Statistically significant differences were observed between genotypes for $\mathrm{Na}^{+}, \mathrm{K}^{+}, \mathrm{and}^{-}$ accumulation in leaves and stems. The $\mathrm{Ca}^{2+}$ and $\mathrm{Mg}^{2+}$ content did not show much variation among genotypes (data not shown). Under salt stress, $\mathrm{Na}^{+}$is mainly accumulated in leaves rather than the stems. Its accumulation in leaves ranged from 28.8 to $46.4 \mathrm{mg}$ per gram of dried samples (Fig. 2a). Leaf $\mathrm{Na}^{+}$content was lower in WRKY13-2, WRKY13-3 RNAi lines and MM. In stems, WRKY12-3, WRKY13-1, WRKY13-2, and WRKY13-3 independent transgenic lines had lower $\mathrm{Na}^{+}$accumulation while WRKY5-3_RNAi line had higher $\mathrm{Na}^{+}$content compared to MM and other lines (Fig. 2a).

Under control condition, $\mathrm{K}^{+}$content was much higher in stems though substantial accumulation of $\mathrm{K}^{+}$was observed in leaves as well (Fig. 2b). Potassium content tended to decrease in stems and leaves of all the genotypes under $100 \mathrm{mM} \mathrm{NaCl}$ treatment. Under salt stress, the highest $\mathrm{K}^{+}$content was observed in stems where $\mathrm{Na}^{+}$was less accumulated. The RNAi plants were slightly different in $\mathrm{K}^{+}$accumulation both in leaves and stems under salt stress. Under salt stress, MM, WRKY12-2, and WRKY13-3 RNAi lines were among the genotypes which had lower $\mathrm{K}^{+}$content in stems, while WRKY5-3 RNAi line had higher $\mathrm{K}^{+}$content compared to MM and other lines.

\section{Expression of Stress Related Genes under Salt Stress}

Based on the morphological, physiological and gene expression data three genotypes (WRKY5-3, WRKY12-2, and WRKY13-3_RNAi lines) including MM were selected for further expression analysis under salt stress. WRKY5-3 had altered phenotype (i.e. necrotic spots) and poor growth performance under control condition that was partly relieved under salt stress. WRKY12-2 and WRKY13-3 RNAi showed good performance both under salt and control conditions; relatively higher shoot length and biomass accumulation with slight variation in leaf $\mathrm{Na}^{+}$content (higher in WRKY12-2 and lower in WRKY13-3). Furthermore, the electrolyte leakage data indicated that WRKY5-3 line was very sensitive to the oxidative stress while WRKY12-2 and WRKY13-3 lines were less sensitive. The variation in growth performance, physiological, and biochemical response of the genotypes might be due to transcriptional regulation of WRKY TFs to various abiotic stress related genes. Thus, the expression of putative abiotic stress related genes involved in hormonal pathways, ROS scavenging, ion homeostasis, NADPHoxidase, and cell death regulation was analyzed in three WRKY_RNAi lines and MM under control and salt stress conditions. 

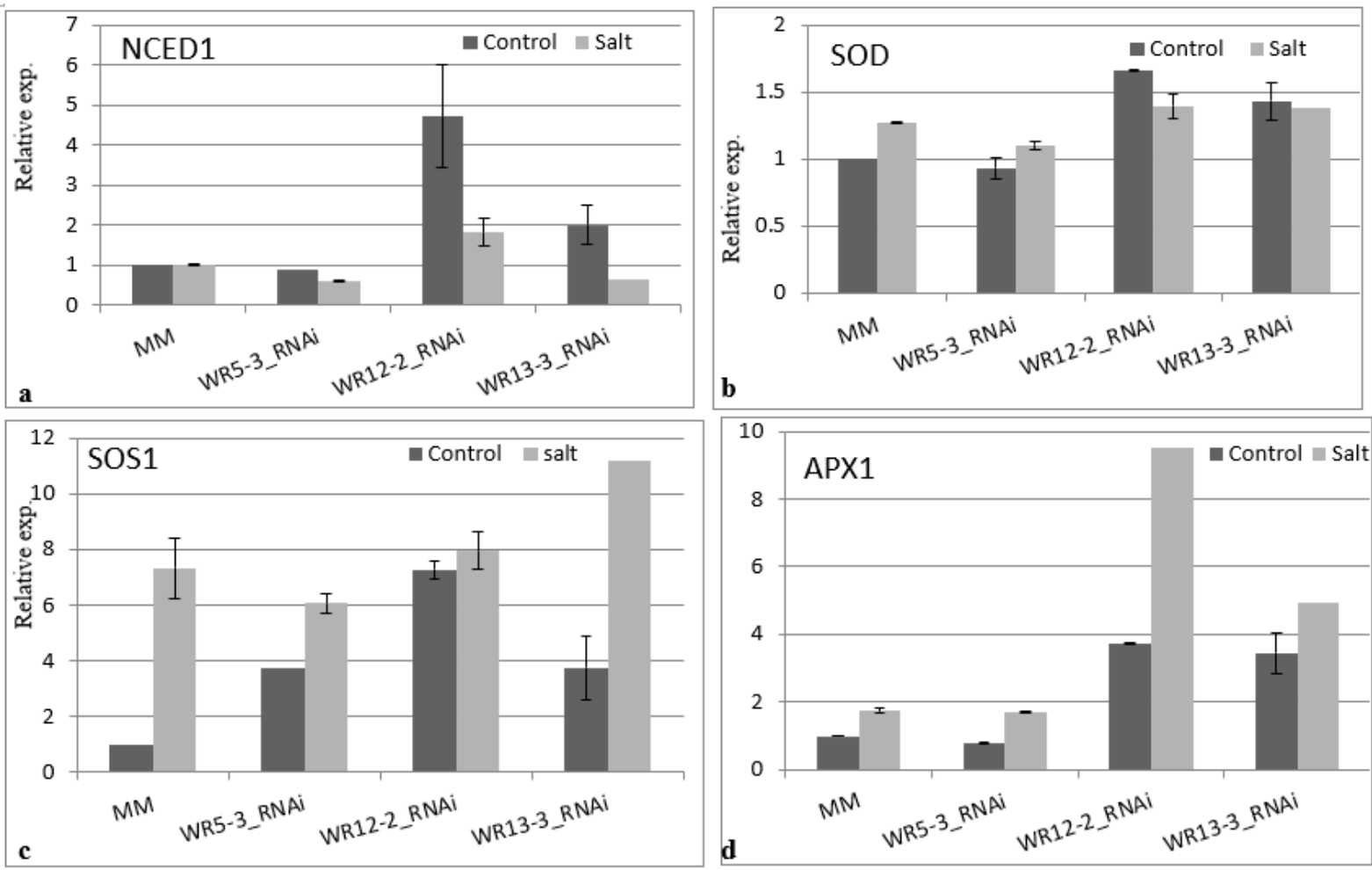

Figure 3. Expression of putative stress related genes in WRKY transgenic and wild type (MM) tomato plants under $100 \mathrm{mM} \mathrm{NaCl}$ and control condition. (a) 9-Cis-epoxycarotenoid Dioxygenase (NCED), (b) Superoxide Dismutase (SOD), (c) Plasma Membrane Na+/H+ Antiporter-1 (SOS1), (d) Ascorbic Peroxide (APX1). Expression value for each gene represent relative to the expression of MM under control condition. Error bars represent standard error.

\section{Abscisic acid (ABA) pathway}

Abscisic acid (ABA) is generally considered as a stress hormone, which is highly induced in response to various biotic and abiotic stresses. Abscisic acid is synthesized from cleavage of carotenoids (9-cis-epoxycarotenoid) by a rate limiting enzyme 9-cis-epoxycarotenoid dioxygenase (NCED) (Aswath et al., 2005). Under salt stress, the expression of NCED1 was slightly higher in WRKY12-2 RNAi compared to other genotypes, while lower in MM, WRKY5-3, and WRKY13-3_RNAi. Under control condition, relatively higher expression was observed in WRKY12-2 and WRKY13-3 RNAi lines compared to MM (Fig.3a).

\section{ROS scavenging genes}

Reactive oxygen species (ROS), generated by different abiotic stresses, are highly toxic and cause damage to proteins, lipids, carbohydrates, DNA, and membrane function (Gill and Tuteja, 2010). Thus, plants have antioxidant response proteins such as superoxide dismutase (SOD), ascorbic peroxide (APX), catalase (CAT) and other non-enzymatic antioxidants. Superoxide dismutase acts as the first defense response under abiotic stresses by removing ROS and producing $\mathrm{H}_{2} \mathrm{O}_{2}$ as a product. Since $\mathrm{H}_{2} \mathrm{O}_{2}$ is still toxic to plant cell, it further detoxified by APX which has high affinity to $\mathrm{H}_{2} \mathrm{O}_{2}$ and play key role in scavenging $\mathrm{H}_{2} \mathrm{O}_{2}$ to $\mathrm{H}_{2} \mathrm{O}$ (Ahmad et al., 2008). Here, the expression of SOD1 and APX1 on selected genotypes both in control and $100 \mathrm{mM} \mathrm{NaCl}$. Under salt stress, expression of SOD was not induced both in transgenic and wild type plants (Fig.3b). Its expression was slightly higher in WRKY12-2 and WRKY13-3 RNAi both under salt and control conditions compared to condition MM and WRKY 5-3 RNAi. On the other hand, the expression of APX1 was induced under salt stress in all transgenic and wild type plants. Moreover, its expression was much higher in WRKY12-2 and WRKY13-3_RNAi under salt and control conditions (Fig.3d), while slightly higher expression detected in MM and WRKY5-3 RNAi under salt stress.

\section{Ion homeostasis}

Under salt stress, plants have different cell transport process that attribute to reduce the accumulation of $\mathrm{Na}^{+}$ions in the cytosol and maintain ion homeostasis. Thus, they can either hinder $\mathrm{Na}^{+}$uptake through utilizing selective ion uptake, sequestrating $\mathrm{Na}^{+}$to the vacuole, or extrusion $\mathrm{Na}^{+}$out of the cell across plasma membrane (Tester and DAVENPORT, 2003). The plasma membrane $\mathrm{Na}^{+} / \mathrm{H}^{+}$antiporter SOS1 (Salt-Over-Sensitive 1) is localized in plasma membrane and proposed to have a role in removal of $\mathrm{Na}+$ from xylem sap and load $\mathrm{Na}^{+}$to the root xylem 
under severe and mild salt stress conditions, respectively (Shi et al., 2002b). It has been also demonstrated that SOS1 involved in $\mathrm{Na}^{+}$partitioning in different plant organs (Olias et al., 2009). In this study, the expression of SOS1 was evaluated in WRKY_RNAi transgenic and wild type plants in response to salt stress. The result shows that salt stress induce the expression of SOS1 in all transgenic and wild type plants, except WRKY12-2 RNAi which showed high expression level under control conditions (Fig.3c). Relative expression of SOS1 was higher in WRKY13-3 RNAi while no differences were observed between MM and WRKY12-2 RNAi under salt.

\section{Regulation of cell death}

Metacaspase (cysteine protease) is involved in regulation of programmed cell death (PCD) in response to biotic and abiotic stresses (Watanabe and Lam, 2011). Here, we checked the expression of metacaspase -1 (MCA1) under control and salt stress condition. As shown in Figure 4, salt stress did not induce the expression of MCA1 in all transgenic and wild type plants. In line with leaf necrotic and cell death phenotype of WRKY5-3_RNAi under control, the highest transcript level of MCA1 was observed in WRKY5-3 RNAi under control condition. Moreover, WRKY12-2 and WRKY13-3 RNAi showed comparatively higher expression of MCA1 under control condition.

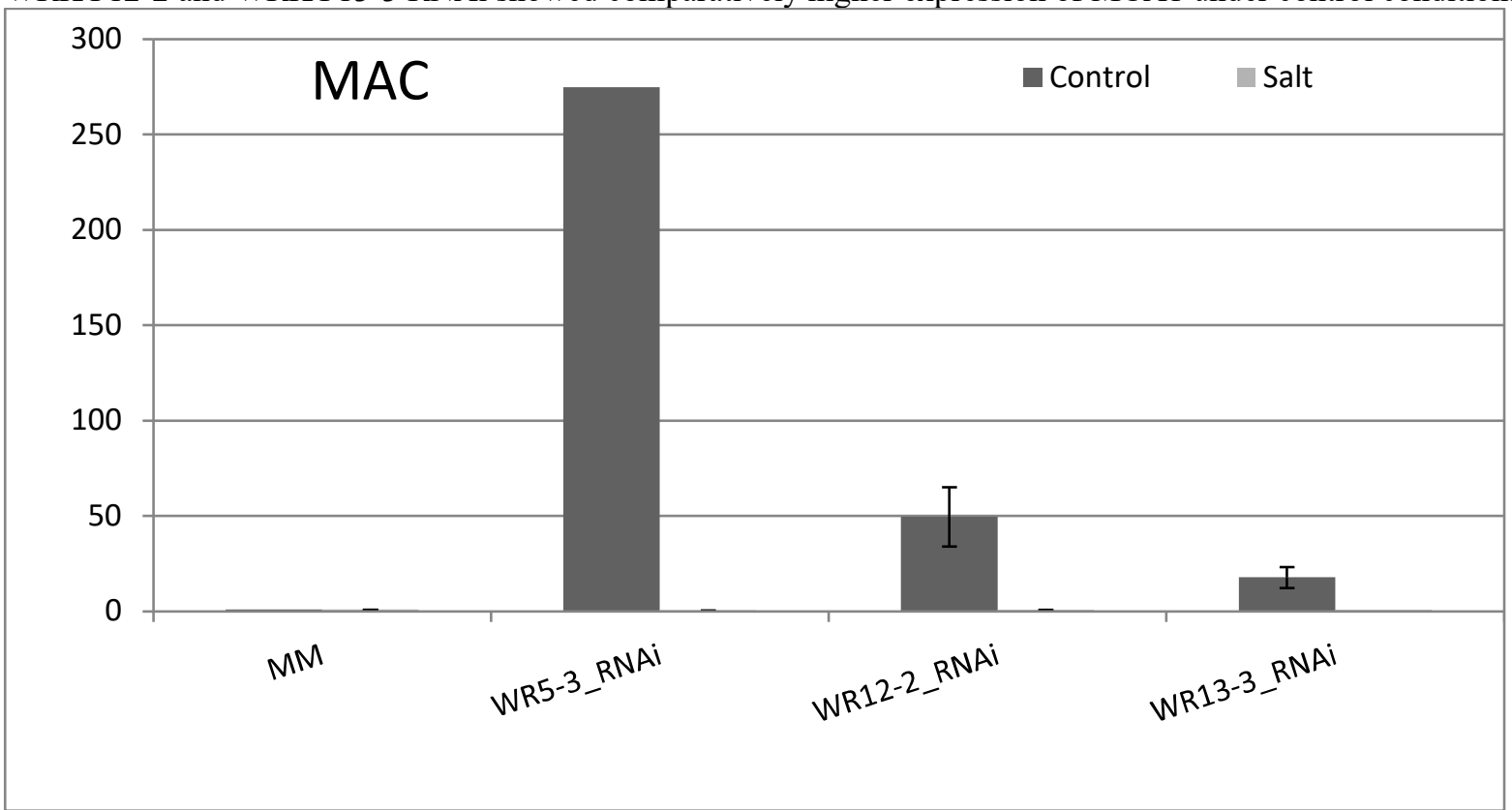

Figure 4. Expression of Metacaspase-1 (MCA1) in WRKY RNAi transgenic and wild type (MM) tomato plants under $100 \mathrm{mM} \mathrm{NaCl}$ and control condition. Expression value for each gene represent relative to the expression of MM under control condition. Error bars represent standard error.

\section{DISCUSSION}

WRKY transcription factors are reported to be involved in negative or positive transcriptional reprogramming associated with biotic and abiotic stresses (Chen et al., 2012; Duan et al., 2014). In this study, the involvement of WRKY genes in salt tolerance was investigated using WRKY RNAi transgenic and wild type (MM) plants under $100 \mathrm{mM} \mathrm{NaCl}$ in comparison to control plants of the respective genotype. Visible phenotypic variation was observed among genotypes after prolonged exposure for salt stress. Though the reduction in biomass due to salinity varies in plant species, the percentage of biomass production in saline compared to control conditions is usually used as indicator for the level of tolerance and sensitivity of plants to salt stress (Greenway and Munns, 1980). Though variation was found among different WRKY transgenes, the results here showed that the shoot length and biomass production were drastically reduced under salt stress compared to control. The reduction of shoot length and biomass might be due to osmotic stress, and/or ion toxicity arising from high $\mathrm{Na}^{+}$in leaves which in turn influences photosynthetic efficiency (Munns, 2002). Previous studies reported that shoot length, photosynthesis, stomatal conductance, plant water status, fresh weight, and dry weight of tomato were adversely affected by increased salt concentration (Hajer et al., 2006; Najla et al., 2007).

In contrary to other studies, the chlorophyll content of the transgenic and wild type plants was increased under salt stress compared to control plants. In another study, however, they argued that salt stress decreased chlorophyll content due to damage of chlorophyll pigment apparatus (Giannakoula and Ilias, 2013) and/or inhibition of chlorophyll biosynthesis resulted from shortage of essential nutrients involved in enzymatic activity. The higher chlorophyll content under salt stress compared to controls could be due to growth retardation (reduction in leaf length and area) which can be faster than chlorophyll degradation leading to increase in chlorophyll concentration 
per unit leaf area under salt stress (Najla et al., 2009). Furthermore, the maintenance of high chlorophyll content under salt stress might be due to the production of anti-oxidant enzymes that prevent the degradation of leaf chlorophyll content (Sevengor et al., 2011).

Interesting phenotypes were observed on lines silenced for WRKY5, WRKY12, and WRKY13. WRKY12-2 and WRKY13-3 RNAi lines performed better both under salt and control conditions with the respective genes being significantly silenced. On the other hand, reduced growth habit and necrotic leaf phenotype was observed for WRKY5-3 RNAi. The chlorophyll content and absolute biomass production was also significantly lower in WRKY5-3 RNAi. WRKY12-2 and WRKY13-3 RNAi transgenic lines had higher shoot length under salt stress and no phenotypic defect (both in salt and control conditions) was observed in independent transgenic lines of the two WRKY genes. Therefore, this suggests that WRKY12 and WRKY13 act as negative regulators in salt stress response. NCBI blasting search was done to find homologous in other plant species having similar functions related to stress response. Three Arabidopsis WRKY TFs (AtWRKY7, AtWRKY11, and WRKY17) which are homologous with tomato WRKY12 (SIWRKY2) are reported as negative regulators in defense response to Pseudomonas syringae (Journot-Catalino et al., 2006; Kim et al., 2006). Similarly, knockout mutants of Arabidopsis AtWRKY27, homologue with WRKY5, showed delayed symptom in response to bacterial pathogen Ralstonia solanacearum (Mukhtar et al., 2008) confirming negative regulation of WRKY5 in defense response. Although several homologous were found in Arabidopsis and other plant species less information is available on the function of WRKYs in abiotic stress response.

In addition to biotic and abiotic stress response, WRKY TF are also involved in plant growth and development (Johnson et al., 2002; Robatzek and Somssich, 2002). Rice OsWRKY78, for instance, is actively involved in stem elongation (Zhang et al., 2011). The higher shoot length of WRKY12-2 and WRKY13-3 RNAi might be due to the release of suppression effect of gibberellins though it needs to be verified further. Although not a homologoues with the studied tomato WRKY genes, OsWRKY71 in rice has been shown as a negative regulator of gibberellins by repressing GA-induced Amy32b a-amylase promoter confirming the negative regulation of a WRKY TF in gibberellins (Zhang et al., 2004).

Salinity disturbs ion homeostasis which affects membrane function, metabolic activity, and growth in plants. To prevent ion toxicity plants have different response mechanisms including selective ion uptake, ion exclusion, and/or vacuolar compartmentation (Tester and DAVENPORT, 2003). Though WRKY12-2 and WRKY13-3 RNAi were found to exhibit greater tolerance to salt stress in terms of growth performance, the tolerance mechanism of the two genotypes might be different. This is because WRKY13-3 RNAi accumulates lower leaf and stem $\mathrm{Na}^{+}$ content, while WRKY12-2 RNAi had significantly higher stem $\mathrm{Na}^{+}$content but, no difference in $\mathrm{K}^{+}$and leaf $\mathrm{Na}^{+}$ content. To have further insight to the molecular basis of the two genotypes, the expression of plasma membrane $\mathrm{Na}^{+} / \mathrm{H}^{+}$antiporter SOS1 (Salt Over Sensitive 1) gene was analyzed in silenced and wild type plants both in control and salt stress. Previously, SOS1 RNAi silenced transgenic tomato plants showed lower growth performance with higher accumulation of $\mathrm{Na}^{+}$ion in roots and leaves under salt stress, while the wild type plants accumulated higher $\mathrm{Na}^{+}$in stems and lower in leaves and roots (Olias et al., 2009). Thus, SOS1 plays critical role in $\mathrm{Na}^{+}$extruding from the roots and partitioning of $\mathrm{Na}^{+}$in different plant organs. Salt stress induced expression of SOS1 both in transgenic and MM plants. Moreover, its expression was highly induced in WRKY13-3 RNAi under salt stress. Thus, the lower accumulation of $\mathrm{Na}^{+}$in leaves and stems of WRKY13-3 RNAi is possibly $\mathrm{Na}^{+}$extruded from the roots to the growth medium. On the other hand, almost the same expression of SOS1 was detected in WRKY122 RNAi under stress and control conditions. Ion content was determined at the whole plant level. Thus, the higher leaf $\mathrm{Na}^{+}$content in WRKY12-2 RNAi does not necessarily entail ion toxicity since the absorbed $\mathrm{Na}^{+}$possibly accumulated in older leaves and/or sequestrated to the vacuoles. Salt tolerant plants maintain high $\mathrm{Na}^{+}$ion concentration in older leaves while lower $\mathrm{Na}^{+}$concentration in young leaves (Cuartero and Fernández-Muñoz, 1998). Plants also have cell transport mechanisms to reduce $\mathrm{Na}^{+}$toxicity and osmotic stress through sequestrating $\mathrm{Na}^{+}$to the vacuole and preventing $\mathrm{Na}^{+}$accumulation in the cytosol (Apse and Blumwald, 2007). Vacuolar NHXtype $\mathrm{Na}^{+} / \mathrm{H}^{+}$antiporters are involved in mediating $\mathrm{Na}^{+}$transport from cytosol to vacuole helped with $\mathrm{H}^{+}$_ATPase and $\mathrm{H}^{+}$PPase producing electrochemical $\mathrm{H}^{+}$concentration gradient (Yarra et al., 2012b). Thus, this suggests that tolerance mechanisms of WRKY12-2 RNAi may be associated with transcriptional regulation of vacuolar $\mathrm{Na}^{+} / \mathrm{H}^{+}$ antiporter genes and this need to be verified further.

Abiotic stresses including salinity induced overproduction of ROS causing disruption of normal metabolic process, damage to cellular structure and enhance oxidative stresses (Ahmad et al., 2008). Hence, measurement of electrolyte leakage (EL), malondialdehyde (MDA) content, and peroxidase activity are most important indicators for the extent of membrane damage and defense response of plants against oxidative stress (Li et al., 2012). Significantly higher EL\% was measured in WRKY5-3 RNAi line both at 24 and 48hrs, while WRKY12-2, WRKY13-3 RNAi lines and MM showed low EL\% both at 24 and 48hrs. The higher EL in WRKY5-3 RNAi may not completely show its sensitivity to oxidative stress. This is because the cell death regulatory gene (MCA1) was up regulated in WRKY5-3 RNAi and this gene might induce high ROS production which already damage the membrane and result in high EL. Furthermore, EL alone wouldn't entail sensitivity and tolerance to oxidative 
stress. It should also be considered that the ROS can be generated from different organelles including mitochondria and leaf peroxisomes besides to chloroplast (Mittova et al., 2003). Bearing this in mind, the variation in EL among the genotypes may be due to enhancement of expression and activities of ROS scavenging genes. WRKY TFs were shown to regulate anti-oxidant defense response through reducing $\mathrm{H}_{2} \mathrm{O}_{2}$ and enhancing peroxidase activity (Miao et al., 2004; Zheng et al., 2013). To confirm expression of anti-oxidant genes, superoxide dismutase (SOD) and ascorbate peroxidase-1 (APX1) were analyzed in transgenic and MM plants. Expression of SOD was slightly higher in WRKY12-2 and WRKY13-3 RNAi both in stress and control conditions. It was shown that SIWRKY overexpressed transgenic tobacco plants accumulated significantly lower MDA, while the POD and SOD level were significantly up-regulated after salt treatment as compared to wild type plants ( $\mathrm{Li}$ et al., 2012). In another study, suppression of ROS accumulation and enhanced activities of ROS-scavenging enzymes (POD, SOD, CAT) were observed in DgWRKY1-overexpresed tobacco plants under salt stress compared to the wild type plants (Liu et al., 2013). In this study, the expression of SOD was slightly higher in WRKY12-2 and WRKY13-3 RNAi lines relative to MM, while lower expression observed in WRKY5-3 RNAi. Similarly, APX1 was highly expressed in WRKY12-2 and WRKY13-3 RNAi, while slightly higher expression observed in WRKY5-3 RNAi both under salt and control conditions. From this result it seems silencing of WRKY12 and WRKY13 may up-regulate the expression of ROS anti-oxidant SOD and APX1 genes, while silencing of WRKY5 down-regulates anti-oxidant genes. Nevertheless, the lower SOD and APX1 in WRKY5-3 may also be related to senescence of the leaf since the activity of SOD and other anti-oxidant enzymes decreased as plants senesce (Mittler, 2002). Moreover, it has to be considered that scavenging and production of ROS is a complex mechanism in which some anti-oxidant enzymes have functional redundancy. WRKY5-3-RNAi showed higher expression of MCA1 which is associated with programmed cell death. Taking all together, the necrotic and yellowish leaf phenotype of WRKY5-3.RNAi might be related to program cell death induced by higher expression of MCA1 and/or ROS production as signaling molecule.

\section{CONCLUSION}

This preliminary result shows that WRKY12 and WRKY13.RNAi lines performed better under salt stress with successful silencing which suggest that the two WRKY TFs act as negative regulators in salt stress tolerance in tomato. Furthermore, there was no fitness cost in these genotypes hence used potentially for breeding. WRKY12 and WRKY13.RNAi showed slight differences in $\mathrm{Na}^{+}$content compared to MM. Furthermore, SOS1 is up regulated in WRKY13_RNAi, which may lead to low $\mathrm{Na}^{+}$accumulation and contribute to salt tolerance. Under salt stress expression of APX was up regulated in WRKY12 and WRKY13.RNAi, which may contribute to the tolerance mechanism of the two genotypes to salt stress. WRKY5 potentially is a negative regulator of cell death, as its silencing resulted in necrotic lesion, increased membrane leakage and increased expression of apoptosis marker genes. Microarray analysis of abiotic stress related genes might give clear indication for the regulatory role of WRKY12 and WRKY13 in abiotic stress tolerance.

\section{Acknowledgment}

This work was supported by Netherlands Fellowship Program (NFP) - Nuffic, Netherlands

\section{REFERENCES}

- $\quad$ Abe H, Urao T, Ito T, Seki M, Shinozaki K, Yamaguchi-Shinozaki K (2003). Arabidopsis AtMYC2 (bHLH) and AtMYB2 (MYB) function as transcriptional activators in abscisic acid signaling. The Plant Cell Online, 15: 63-78.

- Ahmad P, Sarwat M, Sharma S (2008). Reactive oxygen species, antioxidants and signaling in plants. Journal of Plant Biology, 51: 167-173.

- $\quad$ Apse MP, Blumwald E (2007) $\mathrm{Na}^{+}$transport in plants. FEBS letters 581: 2247-2254.

- Aswath CR, Kim SH, Mo SY, Kim DH (2005). Transgenic plants of creeping bent grass harboring the stress inducible gene, 9-cis-epoxycarotenoid dioxygenase, are highly tolerant to drought and $\mathrm{NaCl}$ stress. Plant Growth Regulation, 47: 129-139.

- Besseau, S, Li J, Palva ET (2012). WRKY54 and WRKY70 co-operate as negative regulators of leaf senescence in Arabidopsis thaliana. Journal of Experimental Botany, 63: 2667-2679.

- Bus JS, Gibson JE (1984) Paraquat: model for oxidant-initiated toxicity. Environmental Health Perspectives, 55: 37.

- $\quad$ Chen L, Song Y, Li S, Zhang L, Zou C, Yu D (2012). The role of WRKY transcription factors in plant abiotic stresses. Biochimica et Biophysica Acta (BBA)-Gene Regulatory Mechanisms, 1819: 120-128.

- Cuartero J, Fernández-Muñoz R (1998). Tomato and salinity. Scientia Horticulturae, 78: 83-125.

- Duan GF, Li LJ, Liu QL (2014). A WRKY transcription factor from Malus domestica negatively regulates dehydration stress in transgenic Arabidopsis. Acta Physiologiae Plantarum, 36: 541-548. 
- Eulgem T, Rushton PJ, Robatzek S, Somssich IE (2000). The WRKY superfamily of plant transcription factors. Trends in Plant Science, 5: 199-206.

- Gao JP, Chao DY, Lin HX (2007). Understanding abiotic stress tolerance mechanisms: recent studies on stress response in rice. Journal of Integrative Plant Biology 49, 742-750.

- Giannakoula AE, Ilias I (2013). The effect of water stress and salinity on growth and physiology of tomato (Lycopersicon esculentum Mil.). Archives of Biological Sciences, 65: 611-620.

- Gill SS, Tuteja N (2010). Reactive oxygen species and antioxidant machinery in abiotic stress tolerance in crop plants. Plant Physiology and Biochemistry, 48: 909-930.

- Greenway H, Munns R (1980). Mechanisms of salt tolerance in nonhalophytes. Annual review of plant physiology, 31: 149-190.

- Hajer AS, Malibari AA, Al-Zahrani HS, Almaghrabi OA (2006). Responses of three tomato cultivars to sea water salinity 1 . Effect of salinity on the seedling growth. African Journal of Biotechnology, 5.

- Hannon GJ (2002). RNA interference. Nature, 418: 244-251.

- $\quad \mathrm{Hu}$ H, Xiong L (2014). Genetic engineering and breeding of drought-resistant crops. Annual Review of Plant Biology, 65: 715- 741

- Huang S, Gao Y, Liu J, Peng X, Niu X, Fei Z, Cao S, Liu Y (2012). Genome-wide analysis of WRKY transcription factors in Solanum lycopersicum. Molecular Genetics and Genomics, 287: 495-513.

- Johnson CS, Kolevski B, Smyth DR (2002). TRANSPARENT TESTA GLABRA2, a trichome and seed coat development gene of Arabidopsis, encodes a WRKY transcription factor. The Plant Cell Online, 14: 13591375.

- Journot-Catalino N, Somssich IE, Roby D, Kroj T (2006). The transcription factors WRKY11 and WRKY17 act as negative regulators of basal resistance in Arabidopsis thaliana. The Plant Cell, 18: 3289-3302.

- Kim KC, Fan B, Chen Z (2006). Pathogen-induced Arabidopsis WRKY7 is a transcriptional repressor and enhances plant susceptibility to Pseudomonas syringae. Plant Physiology, 142: 1180-1192.

- $\quad$ Li Jb, Luan YS, Jin H (2012). The tomato SlWRKY gene plays an important role in the regulation of defense responses in tobacco. Biochemical and Biophysical Research Communications, 427: 671-676.

- Liu QL, Xu KD, Pan YZ, Jiang BB, Liu GL, Jia Y, Zhang HQ (2013). Functional Analysis of a Novel Chrysanthemum WRKY Transcription Factor Gene Involved in Salt Tolerance. Plant Molecular Biology Reporter, 1-8.

- Livak KJ, Schmittgen TD (2001). Analysis of Relative Gene Expression Data Using Real-Time Quantitative PCR and the $2^{-\Delta \Delta C T}$ Method. Methods, 25: 402-408.

- Miao Y, Laun T, Zimmermann P, Zentgraf U (2004). Targets of the WRKY53 transcription factor and its role during leaf senescence in Arabidopsis. Plant Molecular Biology, 55: 853-867.

- Mittler R (2002). Oxidative stress, antioxidants and stress tolerance. Trends in Plant Science, 7: 405-410.

- Mittova V, Tal M, Volokita M, Guy M (2003). Up-regulation of the leaf mitochondrial and peroxisomal antioxidative systems in response to salt-induced oxidative stress in the wild salt-tolerant tomato species Lycopersicon pennellii. Plant, Cell \& Environment, 26: 845-856.

- Mukhtar MS, Deslandes L, Auriac MC, Marco Y, Somssich IE (2008). The Arabidopsis transcription factor WRKY27 influences wilt disease symptom development caused by Ralstonia solanacearum. The Plant Journal, 56: $935-947$.

- Munns R (2002). Comparative physiology of salt and water stress. Plant, Cell \& Environment, 25: 239-250.

- Najla S, Vercambre G, Pagès L, Grasselly D, Gautier H, Génard M (2007). Effect of salinity on tomato plant architecture. International Symposium on High Technology for Greenhouse System Management, 801: 11831190 .

- Najla S, Vercambre G, Pagès L, Grasselly D, Gautier H, Génard M (2009) Tomato plant architecture as affected by salinity: descriptive analysis and integration in a 3-D simulation model. Botany, 87: 893-904.

- Olias R, Eljakaoui Z, Li J, DE MORALES PA, MARÍN-MANZANO MC, Pardo JM, Belver A (2009). The plasma membrane $\mathrm{Na}^{+} / \mathrm{H}^{+}$antiporter SOS1 is essential for salt tolerance in tomato and affects the partitioning of $\mathrm{Na}^{+}$between plant organs. Plant, Cell \& Environment, 32: 904-916.

- Orellana S, Yanez M, Espinoza A, Verdugo I, Gonzalez E, RUIZ-LARA S, Casaretto JA (2010). The transcription factor SlAREB1 confers drought, salt stress tolerance and regulates biotic and abiotic stressrelated genes in tomato. Plant, Cell \& Environment, 33: 2191-2208.

- Ramamoorthy R, Jiang SY, Kumar N, Venkatesh PN, Ramachandran S (2008). A comprehensive transcriptional profiling of the WRKY gene family in rice under various abiotic and phytohormone treatments. Plant and Cell Physiology, 49: 865-879.

- $\quad$ Robatzek S, Somssich IE (2002). Targets of AtWRKY6 regulation during plant senescence and pathogen defense. Genes \& Development, 16: 1139-1149. 
- $\quad$ Ross CA, Liu Y, Shen QJ (2007). The WRKY Gene Family in Rice (Oryza sativa). Journal of Integrative Plant Biology, 49: 827-842.

- Sevengor S, Yasar F, Kusvuran S, Ellialtioglu S (2011). The effect of salt stress on growth, chlorophyll content, lipid peroxidation and antioxidative enzymes of pumpkin seedling. African Journal of Agricultural Research, 6: 4920-4924.

- $\quad$ Shi H, Quintero FJ, Pardo JM, Zhu JK (2002b). The putative plasma membrane $\mathrm{Na}^{+} / \mathrm{H}^{+}$antiporter SOS1 controls long-distance $\mathrm{Na}^{+}$transport in plants. The Plant Cell Online, 14: 465-477.

- $\quad$ Singh KB, Foley RC, Oñate-Sánchez L (2002). Transcription factors in plant defense and stress responses. Current Opinion in Plant Biology, 5: 430-436.

- $\quad$ Tester M, Davenport R (2003). $\mathrm{Na}^{+}$tolerance and $\mathrm{Na}^{+}$transport in higher plants. Annals of Botany, 91: 503527.

- $\quad$ Ülker B, Somssich IE (2004). WRKY transcription factors: from DNA binding towards biological function. Current Opinion in Plant Biology, 7: 491-498.

- Watanabe N, Lam E (2011). Arabidopsis metacaspase $2 \mathrm{~d}$ is a positive mediator of cell death induced during biotic and abiotic stresses. The Plant Journal, 66: 969-982.

- Wu C, Niu Z, Tang Q, Huang W (2008). Estimating chlorophyll content from hyperspectral vegetation indices: Modeling and validation. Agricultural and Forest Meteorology, 148: 1230-1241.

- Yarra R, He SJ, Abbagani S, Ma B, Bulle M, Zhang WK (2012b). Overexpression of a wheat $\mathrm{Na}^{+} / \mathrm{H}^{+}$antiporter gene $\left(\mathrm{TaNHX}_{2}\right)$ enhances tolerance to salt stress in transgenic tomato plants (Solanum lycopersicum L.). Plant Cell, Tissue and Organ Culture, 111: 49-57.

- Zhang CQ, Xu Y, Lu Y, Yu HX, Gu MH, Liu QQ (2011). The WRKY transcription factor OsWRKY78 regulates stem elongation and seed development in rice. Planta, 234: 541-554.

- Zhang ZL, Xie Z, Zou X, Casaretto J, Ho T-hD, Shen QJ (2004). A rice WRKY gene encodes a transcriptional repressor of the gibberellin signaling pathway in aleurone cells. Plant Physiology, 134: 1500-1513.

- Zheng L, Liu G, Meng X, Liu Y, Ji X, Li Y, Nie X, Wang Y (2013). A WRKY gene from Tamarix hispida, ThWRKY4, mediates abiotic stress responses by modulating reactive oxygen species and expression of stressresponsive genes. Plant Molecular Biology, 82: 303-320.

- Zheng X, Chen B, Lu G, Han B (2009). Overexpression of a NAC transcription factor enhances rice drought and salt tolerance. Biochemical and Biophysical Research Communications, 379: 985-989.

- Zhou QY, Tian AG, Zou HF, Xie ZM, Lei G, Huang J, Wang CM, Wang HW, Zhang JS, Chen SY (2008). Soybean WRKY-type transcription factor genes, GmWRKY13, GmWRKY21, and GmWRKY54, confer differential tolerance to abiotic stresses in transgenic Arabidopsis plants. Plant Biotechnology Journal, 6: 486503 . 